\title{
Design and Control Considerations for a Wave Energy Converter
}

\author{
Gary Nolan and John Ringwood \\ Department of Electrical Engineering \\ National University of Ireland, Maynooth \\ Ireland
}

E-mail: gary.nolan@eeng.may.ie john.ringwood@eeng.may.ie

\begin{abstract}
This paper discusses the main power take-off design issues and the resulting control strategy of a wave energy converter with the dual function of producing potable water and generating electricity.

Keywords - Wave Energy, Power Take-Off Design, Reverse Osmosis.
\end{abstract}

\section{INTRODUCTION}

A wave energy converter (WEC) is a device that converts the energy of ocean surface waves into useful forms of energy. There are many difficulties to overcome in achieving this, such as the harsh environments they operate in, the randomness in the power input of the waves and the large loads experienced in extreme weather conditions. These are some of the reasons why there are only a number of full scale prototype devices around the world. The type of device this paper considers is an offshore device that captures the energy in their heave or rotational motion, caused by the sea waves, using a hydraulic power take-off system. Examples of these include heaving buoy devices, such as Wavebob, and hinge-barge devices, such as the McCabe Wave Pump (MWP) [1] and the Pelamis [2]. Captured energy is in the form of fluid under pressure, which can be used to either generate electricity, using a turbine and generator, or to produce potable drinking water from seawater by reverse osmosis (RO). RO is a desalination technique that purifies water under pressure using a semi-permeable membrane. The membrane allows water particles to be passed through it while rejecting any impurities. While the actual energy capture technique of the WEC remains the same for each of the output cases (potable water production and electricity generation) the design and control of the power take-off (PTO) system is different.

This paper considers whether a single device can produce both potable water and electricity side by side. In this scenario a number of conflicting objectives arise. The paper will consider the limitations each system puts on the other and the trade-offs that have to be made to realise a dual function machine. It will conclude by documenting whether it is possible to have a dual function machine that can produce significant quantities of potable water and electricity together and whether there are any advantages in doing so.

The paper will begin by giving a problem specification, followed by a description of a full WEC model. The main design issues are then discussed, the control system formalised and conclusions made.

\section{Problem Specification}

The problem specification, for designing a dual function PTO system, is to maximise the energy conversion from the sea waves in terms of producing potable water and generating electricity, while maintaining the structurial integrity of the device. This can be written as the performance criterion,

Maximise:

$P_{f}=\int_{t=0}^{t_{1}}\left(\alpha Q_{w}(t)+\beta k P_{g}(t)\right) d t$

subject to:

$|\zeta|<\zeta(\max ),|\dot{\zeta}|<\dot{\zeta}(\max ),|\ddot{\zeta}|<\ddot{\zeta}(\max )$

where $\alpha$ and $\beta$ are user specified weightings, $Q_{w}$ is the flow of potable water, $P_{g}$ is the power generated into the grid, $k$ is a unit balancing constant, $\zeta$ is the vertical (heave) displacement and $t_{1}$ is a fixed time period such as a day, a week or a year.

\section{Wave Energy Converter Model}

Figure 1 represents a generalised WEC (including the PTO system) in block diagram form, which can be broken down into three areas as follows,

1. The mechanical rig and its corresponding equations of motion, 


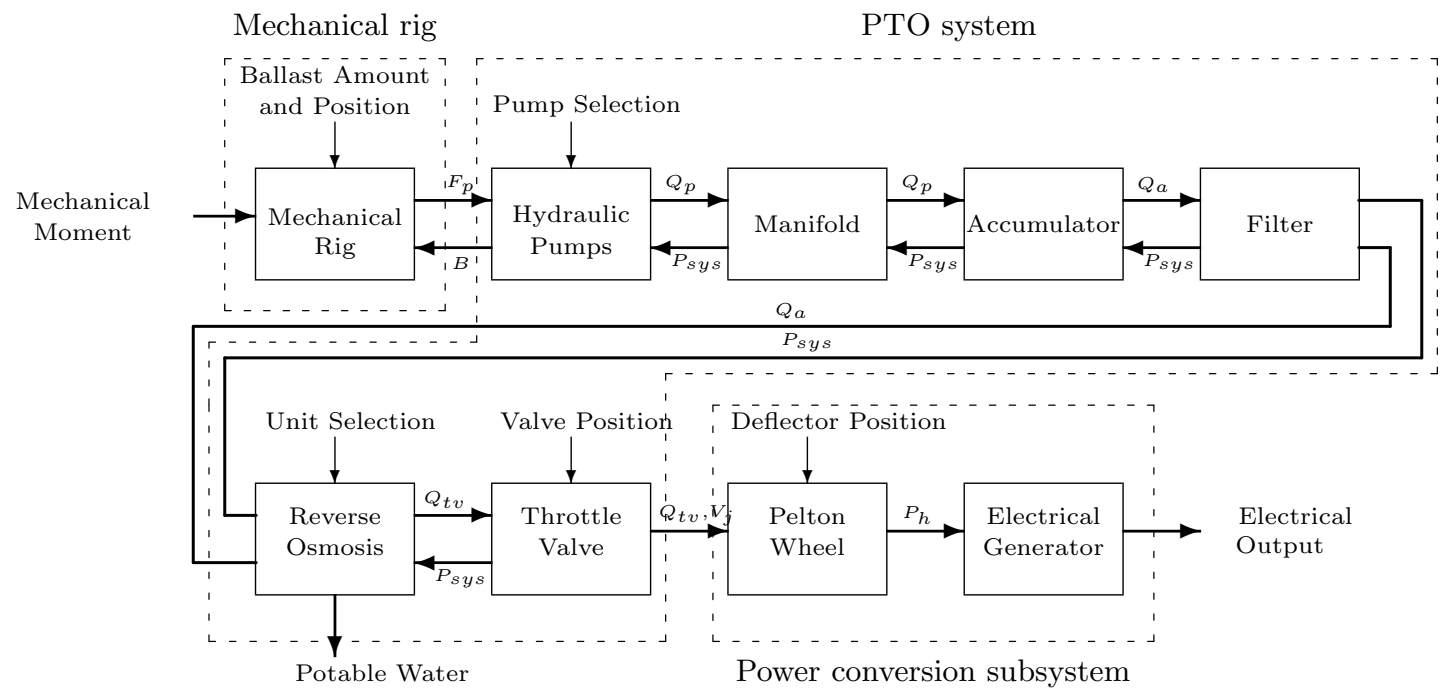

Fig. 1: Block Diagram of a Complete WEC

2. The power take-off system (including the RO subsystem), and

3. The power conversion subsystem.

\section{a) Equations of Motion}

Only the equations of motion for a point absorber are dealt with in this section. These have been chosen ahead of others due to their relative simplicity. However, the equations of motion for other devices can be found. For example, the equations of motion for a hinge-barge device have been developed by Kraemer and can be found in [1].

A conceptual representation of a point absorber is depicted in figure 2. It consists of a cylindrical device which is constrained to move in a vertical direction only. The dynamics of the device can be

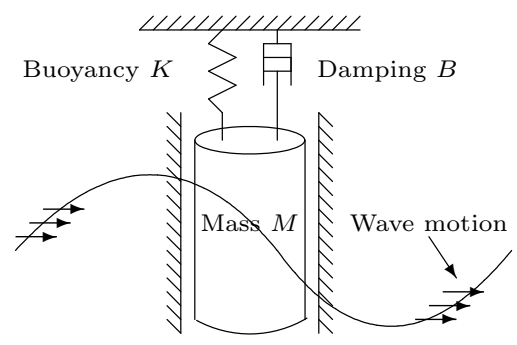

Fig. 2: Conceptual point absorber

approximately described by the following integrodifferential equation, given by Barbarit et al [3],

$$
\begin{aligned}
& \left(M+\mu_{\infty}\right) \ddot{\zeta}(t)+\int_{0}^{t} \dot{\zeta}(t) k(t-\tau) d \tau+ \\
& B \dot{\zeta}(t)+K \zeta(t)=F(t)
\end{aligned}
$$

where $F(t)$ is the heave force due to the incident waves, $\mu_{\infty}$ is the instantaneous added mass and $\int_{0}^{t} \dot{\zeta}(t) k(t-\tau) d \tau$ is a memory term of the wave radiation forces. The mass term, $M$, can include moveable ballast.

As illustrated in figure 1 there is an exchange of driving and damping forces between the mechanical rig and the PTO system via the hydraulic pumps. This is reflected in the WEC's equation of motion through the damping term, $B$, which is calculated from, the pressure across the pump chambers, $P_{\text {sys }}$, and the flow from the pump, $Q_{p}$, using the following equation:

$B=\frac{A^{2} P_{s y s}}{Q_{p}}$

where $\mathrm{A}$ is the pump area in $m^{2}$.

\section{b) Power Take-Off and Power Conversion Sys- tem Models}

These authors have modelled a PTO system for a hinge-barge WEC in a previous paper [4]. The model was created using bondgraph modelling [5], which is based on the concept of power flow modelling. Although this model is specific to the PTO system of a hinge-barge device it can be easily modified to represent a PTO system of a point absorber. The basics of the model are as follows, with the bondgraphs tieing the various parts of the model toegether.

The hydraulic pumps are modelled as a single mechanical-hydraulic transformer, as given by Ljung [6], and convert the driving force, $F(t)$, from the devices equation of motions into a corresponding pressure, $P_{s y s}$, and flow, $Q_{p}$ using equations 
(5) and (6).

$P_{\text {sys }}=F / A$

$Q_{p}=v / A$

where $v$ is the velocity of the pump.

The manifold sums the flows from the various pumps. The accumulator is modelled as a capacitive element, relating its change in pressure, $\triangle p_{a c c}$, with its change in volume, $\triangle v_{a c c},[7]$ by:

$\triangle p_{a c c}=\left(p_{0} \gamma / v_{0}\right) \triangle v_{a c c}$

giving the capacitance value, $C_{a c c}$, as:

$C_{a c c}=\left(v_{0} / p_{0} \gamma\right)$

where $v_{o}$ and $p_{o}$ are the accumulators precharge volume and pressure respectively and $\gamma$ is the ratio of specific heats of the gas at a constant temperature and a constant volume.

The water filter is simply modelled as a series resistance, with its only effect on the PTO variables being a loss in pressure. The membranes of the RO units and the throttle valve are also modelled as resistive elements. The RO membrane resistance is calculated from its pressure-flow characteristic, illustrated in figure 3 . The figure highlights the osmotic pressure, $P_{\text {osm }}$, which must be exceeded before potable water is produced and also illustrates the limit on the working pressure of the RO units. Continuously exceeding this limit will result in a drastic reduction of the membrane lifetime. By adding RO membrane resistances in parallel,

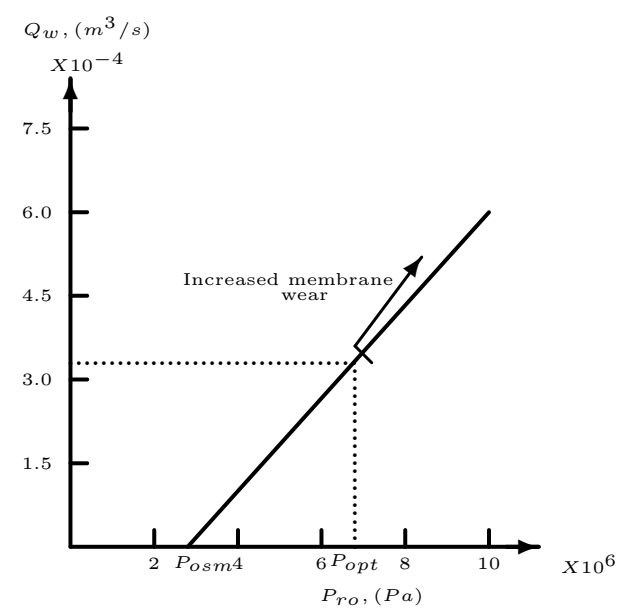

Fig. 3: Pressure-Flow Characteristic for a single RO unit

the switching in and out of multiple banks of RO membranes is modelled. The model of the RO unit is completed by noting there is a minimum limit on the ratio of potable water flow, $Q_{w}$, to flow out through the throttle valve, $Q_{t v}$, as:

$\frac{Q_{w}}{Q_{t v}}<\frac{1}{9}$
When potable water is produced, the salt concentration in the remaining volume of water is increased, increasing the osmotic pressure and hence reducing the flow of potable water. For this reason, there needs to be a sufficient $Q_{t v}$ to pass on the highly concentrated flow and keep the osmotic pressure down.

The resistance, $R_{t v}$, representing the throttle valve is given by a combination of equation (10) [8] and the inherent flow characteristic of the valve, as illustrated in figure 4.

$Q_{t v}=C_{v} \sqrt{P_{s y s}}$

giving,

$R_{t v}=\frac{d P_{s y s}}{d Q_{t v}}=\frac{\sqrt{P_{s y s}}}{0.5 C_{v}}$

where, $C_{v}$ is the valves rated flow coefficient.

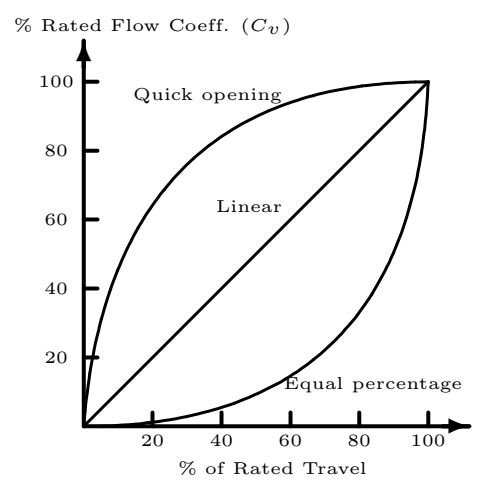

Fig. 4: Throttle Valve Inherent flow Characteristic

A Pelton wheel is the ideal turbine for electricity generation in this PTO system, due to the fact that it is a high pressure low flow machine which matches the fluid energy produced by the WEC. It is modelled by a number of equations and an efficiency curve. The equation for the hydraulic power, $P_{h}$, delivered to the Pelton wheel by the jet of fluid is given [9] by:

$P_{h}=(1 / 2) \rho V_{j}^{2} Q_{t v}$

with,

$V_{j}=\sqrt{2\left(P_{\text {sys }} / \rho\right)}$

where $\rho$ is the fluid density and $V_{j}$ is the jet velocity. Figure 5(a) shows the variation of the Pelton wheel efficiency, $\eta_{p w}$, with the speed ratio of the wheel velocity, $U$, to the jet velocity, where:

$\eta_{p w}=P_{m e c h} / P_{h}$

with,

$P_{m e c h}=T_{p w} \omega_{p w}$ 


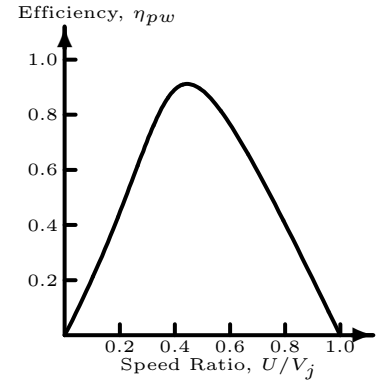

(a)

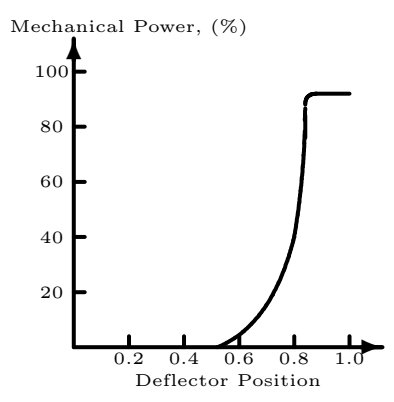

(c)

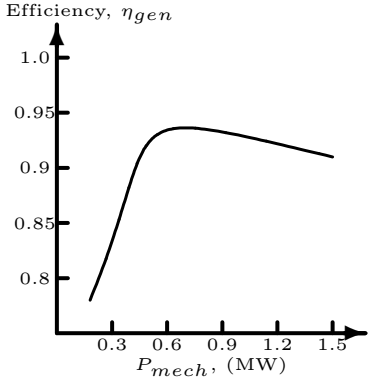

(b)

Fig. 5: (a) Pelton Wheel Efficiency, (b) Deflector Power Curve, (c) Generator Efficiency

where $P_{\text {mech }}$ is the mechanical power of the Pelton wheel, $T_{p w}$ is the torque and $\omega_{p w}$ is the rotation speed. Part (b) of the same figure illustrates the power curve of the Pelton wheel deflector which can be used to deflect part of the water jet from the turbine. The PTO system model is completed by the generator efficiency curve, an example of which is in figure $5(\mathrm{c})$. It is worth noting that the turbine and generator set does not load the rest of the PTO system since there is a physical gap between them and the throttle valve.

\section{Design Considerations}

Designing the PTO system for dual function operation involves designing to maximise the performance function given in equation (17) for the nominal wave amplitudes. This is achieved through a combination of appropriate system physical design and addition of a control system to cater for varying sea conditions. This section focuses on the former.

The PTO systems design variables are,

- Size and number of RO units,

- Rating of Pelton wheel,

- Rating and type of generator, and

- Size and precharge pressure of accumulator.

a) Type of Generator

The first and biggest design decision is the choice of a fixed speed or a variable speed generator. The advantage of variable speed operation is the ability to vary the velocity of the jet of water from the throttle valve so as to actively achieve maximum power to the Pelton wheel for the particular amount of captured fluid energy (eqn. (12)). However, varying the jet velocity requires varying the system pressure (eqn. (13)) and may result in operation of the RO units at non-optimal pressure. Also, variable speed machines require power electronics for an AC-DC-AC conversion and these electronics may effect the reliability (a major issue for offshore devices) and introduce further efficiency degradation. For these reasons a fixed speed generator should be chosen in a dual function machine. This will allow a constant PTO system operating pressure to be maintained, giving a constant pressure in the RO units and a constant jet velocity for the Pelton wheel.

\section{b) $R O$ design}

The PTO system operating pressure is chosen so as to allow the RO units to operate at their most efficient (figure 3). It is chosen at the highest allowable membrane pressure, within the safe operating range (approximately 67 bar for the characteristic in figure 3 ). The size of the individual RO units is chosen by trading off efficiency against cost and control energy. A greater number of small $\mathrm{RO}$ units allows the number of RO units employed to be better matched with the available flow at the max system pressure and hence keeps efficiency high. However, a single unit will have a lower capital cost and there will be no requirement on control energy to switch in and out units. Either way the total size of the RO units should be sufficient to allow maximum potable water flow at nominal wave amplitudes. Extra capacity could be allowed to enable further potable water production at wave amplitudes greater then nominal.

\section{c) Pelton Wheel and Generator Ratings}

Using equation (13), a fixed operating pressure means a constant jet velocity to the Pelton wheel. The power delivered to the Pelton wheel is now only dependant on the flow from the throttle valve (eqn. (12)). The optimum bucket speed, $U$, is given by the efficiency curve of figure 5(a) and is roughly half the jet velocity. The optimum radius of the Pelton wheel, $R$, is then calculated as:

$R=U / \omega_{p w}$

where $\omega_{p w}$ is the rotation speed of the Pelton wheel. This is matched to the rotation speed of 
the generator, $\omega_{g}$, which is dictated by the grid frequency, $f_{g}$, and the number of poles, $N_{p}$, in the machine as follows,

$\omega_{p w} N_{1}=\omega_{g} N_{2}=60 f_{g} / N_{p}$

where $N_{1} / N_{2}$ is the gearing ratio. The size of the electrical generator is chosen to maximise energy out to the grid, $E_{\text {out }}$, as:

$E_{\text {out }}=\int_{t=0}^{t_{1}} \eta_{\text {gen }}\left(P_{\text {in }}\right) P_{\text {in }}(t) d t$

with $P_{\text {in }}$ being the power supplied to the generator after any losses have been subtracted, $\eta_{g e n}$ is the generator efficiency and $t_{1}$ is a fixed time period such as a year.

\section{d) Accumulator Design}

An accumulator is a device for storing energy in the form of fluid under pressure. Due to its fast response time, a gas-loaded bladder type accumulator is most appropriate for this system. Its function is to remove or dampen (as much as possible) the pulsatile nature of the fluid energy captured by the hydraulic pumps. Effective sizing of the accumulator means a lot less work for the throttle valve in maintaining a constant operating pressure and also a much steadier fluid flow to the Pelton wheel. Hydac Technology Corporation [10] recommend, for pulsation dampening,:

$p_{o}=(0.6-0.8) P_{s y s}$

where $p_{o}$ is the precharge pressure of the accumulator and $P_{\text {sys }}$ is the maintained system pressure. The precharge volume, $v_{o}$, should be sufficient to hold the maximum volume deviations for the flow levels at the maintained operating pressure.

\section{e) Overall design}

Using the characteristics and equations given in section III and section IV, a WEC that could generate $1000 \mathrm{~L} / \mathrm{min}$ of flow at a system pressure of 67 bar could produce $100 \mathrm{~L} / \mathrm{min}$ of potable water flow while at the same time generate over a $100 \mathrm{KW}$ of power into the electrical grid system. This may be achievable for a single hinged-barge device but is probably unrealistic for a single point absorber device. However, there is always a possibility of linking the PTO systems of a number of point absorber devices and having one RO system and one generator system for all.

\section{Control Issues}

The WEC control problem is a nonlinear, multivariable problem. The manipulated variables (MVs) are:

- Position of throttle valve,
- Ballast quantity and position,

- Number of pumps employed, and

- Number of RO units employed.

The controlled variables $(\mathrm{CVs})$ are:

- Flow of potable water,

- Throttle valve flow, and

- Displacements (or angles) and velocities of device.

In a device only producing electricity, the throttle valve position is the primary MV, actively controlling the rig dynamics through control of the device damping, $B$. In a point absorber device, the buoyancy, $K$, and mass, $M$, are designed so as to make the resonant frequency of the device, $\omega_{n}$, equal to the nominal wave frequency, $\omega_{w}$, as follows:

$\omega_{n}=\sqrt{K / M}=\omega_{w}$

The profile of the damping, $B$, across the wave period is then chosen so as to optimise the energy captured over the wave cycle. However, in a dual machine this is not possible as the function of the throttle valve is to regulate the system pressure at the designed operating point (reflecting the RO pressure and the water jet velocity needs).

The ballast quantity (in the case of hinged-barge and point absorber device) and position (in the case of hinged-barge device only) allow some control of the barge dynamics through the inertia term, $M$, of equation (3). However, pumping ballast around the device is a slow process requiring a lot of control energy and will only be used when there is a significant change in wave conditions, so as to maximise energy capture or prevent flogging of the device itself. Switching in and out of hydraulic pumps allows some minimal control of the device damping. Switching in an extra pump can be thought of as increasing the pump area in equation (4) and increases the damping. However, it is unlikely that there will be sufficient numbers of pumps to allow accurate control of the damping. The final MV is the number of RO units employed and this is simply determined, using equation (9), so as to have the maximum number of RO units switched in at any particular time.

Overall the control system, excluding the throttle valve control, must switch in and out pumps or RO units depending on predefined operating ranges, as illustrated in figure 6 . This brings the control problem into the realms of switching systems where care needs to be taken to ensure that stability and performance is maintained across switching boundaries [11]. However, the relatively slow dynamics of a WEC permit significant computation and numerical optimisation in the control law. 


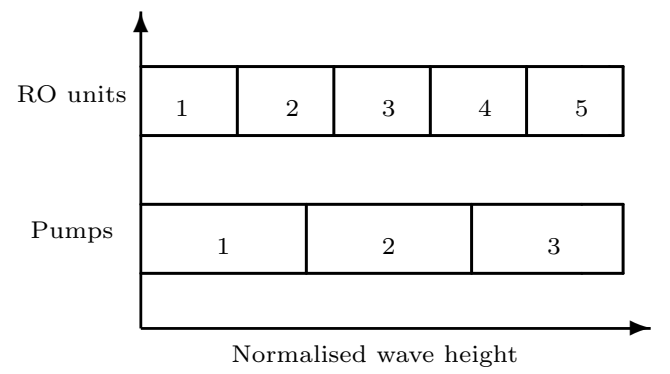

Fig. 6: Control Operating Ranges

\section{Limitations of Dual Design}

In a dual function machine, the electricity generation is not at its most efficient as the fluid jet velocity to the pelton wheel is limited by the maximum operating pressure of the RO membranes. Dual function operation removes the requirement of using a variable speed generator, which would allow active variation of the jet velocity so as to maximise the hydraulic power, (eqn. (12)), delivered to the Pelton wheel.

The dual design requires a constant system pressure to be maintained. This prevents the throttle valve from attempting to maximise the captured energy from the sea waves by trying to control the rig dynamics through control of the damping term, $B$, in equation (3). This is especially the case for a point absorber type device that may attempt to use the PTO system to achieve latching $[3,12]$.

\section{CONCLUSION}

This paper has addressed the possibility of using a single WEC to produce potable water and electricity simultaneously. The paper has two main conclusions. Firstly, there is a conflict between using the throttle valve to control,

- The damping of the rig (to maximise captured energy from the sea), and

- Maintaining constant system pressure (for optimal operation of the RO and Pelton wheel).

Secondly, the facility for electronic computer control is limited since the successful operation of a dual function WEC depends heavily on the mechanical design (eqn. (20)), and the PTO design (section IV). The remaining functions include, the throttle valve control, controlling the moveable ballast, $M$, and actively switching the RO units and the pumps.

\section{ACKNOWLEDGEMENTS}

This research was funded by Enterprise Ireland under the Research Innovation Fund scheme (Project IF/2002/309).

\section{REFERENCES}

[1] D.R.B. Kraemer. "The motions of hingedbarge systems in regular seas.". PhD. Thesis, Johns Hopkins University, 2001.

[2] (-) "Ocean Power Delivery website [online]". Available from: http://www.oceanpd.com, [Accessed Febuary 2003].

[3] A. Babarit, G. Duclos and A.H. Clément. "Comparison of latching control strategies for a heaving wave energy device in random sea". Proc. 5th European Wave Energy Conference, Cork, Ireland, 2003.

[4] G. Nolan, M. Ó Catháin, J. Murtagh and J.V. Ringwood. "Modelling and Simulation of the Power Take-Off System for a Hinge-Barge Wave-Energy Converter". Proc. 5th European Wave Energy Conference, Cork, Ireland, 2003.

[5] A.K. Samantaray. "About bond graphs and application notes [online]". Available from: http://www.bondgraphs.com, [Accessed Febuary 2003].

[6] L. Ljung and T. Glad. "Modeling of Dynamic Systems". Prentice Hall, 1994.

[7] (-) "College of Engineering and Technology, Brigham Young University. Dynamic system modelling lecture notes [online]". Available from: http://class.et.byu.edu/me435-3/, , [Accessed March 2003].

[8] B.E. Larock, R.W. Jeppson \& G.Z. Waters. "Hydraulics of Pipeline Systems". CRC Press, 1999.

[9] (-) "Summary of theory for the Pelton wheel [online]". Available from: http://learn.hw.ac.uk/fluidmech/peltonsummary.pdf, [Accessed March 2003].

[10] Hydac Technology Corporation website. "Accumulators". http://www.hydacusa.com/, [Accessed March 2003].

[11] D. Liberzon and A.S. Morse. "Basic problems in stability and design of switched systems". IEEE Control Systems Magazine, 19, 5, 59-70, 1999.

[12] K. Budal and J. Falnes. "A resonant point absorber of ocean wave power". Nature, 256:478479, 1975. 